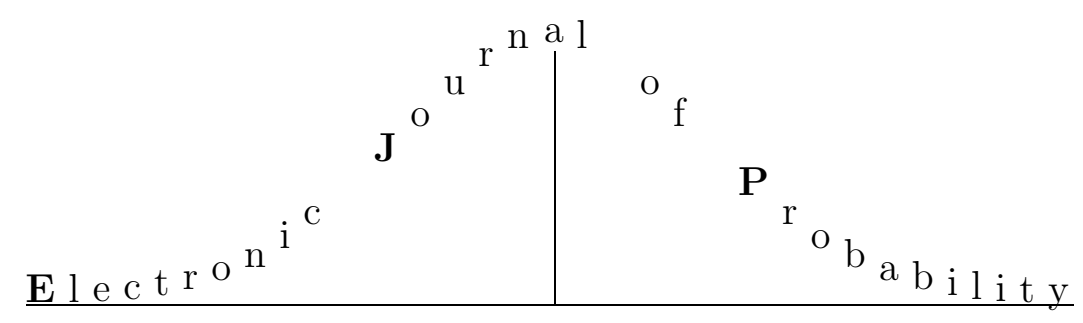

Vol. 10 (2005), Paper no. 31, pages 1005-1025.

Journal URL

http://www.math.washington.edu/ ejpecp/

\title{
Convergence in Dirichlet Law of Certain Stochastic Integrals.
}

\author{
Christophe Chorro \\ Cermsem, Université Paris 1, Maison des sciences économiques, \\ 106-112 Boulevard de l'Hôpital 75647 Paris cedex 13, France \\ E-mail: christophe.chorro@gmail.com
}

\begin{abstract}
Recently, Nicolas Bouleau has proposed an extension of the Donsker's invariance principle in the framework of Dirichlet forms. He proves that an erroneous random walk of i.i.d random variables converges in Dirichlet law toward the Ornstein-Uhlenbeck error structure on the Wiener space [4]. The aim of this paper is to extend this result to some families of stochastic integrals.
\end{abstract}

Keywords: Invariance principle, stochastic integrals, Dirichlet forms, squared field operator, vectorial domain, errors.

AMS Subject Classification (2000): 31C25, 47B25, 49Q12, 60B12, $60 \mathrm{H} 05,60 \mathrm{H} 07$.

Submitted to EJP on April 27, 2005. Final version accepted on June 14, 2005. 


\section{Introduction}

The error calculus, based on the theory of Dirichlet forms ([7],[12],[17]), is a natural extension of the seminal ideas of Gauss concerning small errors and their propagation ([6], chap.1). It gives a powerful framework suitably adapted to the infinite dimensional stochastic models used in finance and physics ([6], chap.7).

From now on, an error structure will be a term $S=(W, \mathcal{W}, P, \mathbb{D}, \Gamma)$ where $(W, \mathcal{W}, P)$ is a probability space, $\mathbb{D}$ is a dense sub-vector space of $L^{2}(W, \mathcal{W}, P)$ (denoted by $L^{2}(P)$ ) and $\Gamma$ is a positive symmetric bilinear map from $\mathbb{D} \times \mathbb{D}$ into $L^{1}(P)$ fulfilling:

1) the functional calculus of class $C^{1} \cap$ Lip. This expression means that if $U \in \mathbb{D}^{n}, V \in \mathbb{D}^{p}$, for all $F \in C^{1}\left(\mathbb{R}^{n}, \mathbb{R}\right) \cap \operatorname{Lip}=\left\{C^{1}\right.$ and Lipschitz $\}$ and $G \in C^{1}\left(\mathbb{R}^{p}, \mathbb{R}\right) \cap$ Lip then $(F(U), G(V)) \in \mathbb{D}^{2}$ and

$$
\Gamma[F(U), G(V)]=\sum_{i, j} F_{i}^{\prime}(U) G_{j}^{\prime}(V) \Gamma\left[U_{i}, V_{j}\right] \quad P \text {-a.e }
$$

2) $1 \in \mathbb{D}$, (thus $\Gamma[1]=0)$

3) the bilinear form $\mathcal{E}[F, G]=\frac{1}{2} \mathbb{E}_{P}[\Gamma[F, G]]\left(\mathbb{E}_{P}[\Gamma[F, G]]=\int_{W} \Gamma[F, G] d P\right)$ defined on $\mathbb{D} \times \mathbb{D}$ is closed i.e $\mathbb{D}$ is complete under the norm of the graph

$$
\|\cdot\|_{\mathbb{D}}=\left(\|\cdot\|_{L^{2}(P)}^{2}+\mathcal{E}[.]\right)^{\frac{1}{2}} .
$$

We will always write $\Gamma[F]$ for $\Gamma[F, F]$ and $\mathcal{E}[F]$ for $\mathcal{E}[F, F]$.

The property (1) is none other than the so-called Gauss' law of small error propagation by regular functions ([6], chap.1). Thus, for $U=\left(U_{1}, \ldots, U_{n}\right) \in$ $\mathbb{D}^{n}$, the intuitive meaning of the matrix $\underset{=}{\Gamma}[U]=\left[\Gamma\left[U_{i}, U_{j}\right]\right]_{1 \leq i, j \leq n}$ will be the conditional covariance matrix of the error on $U$ given $U$.

With the hypotheses mentioned above, the form $\mathcal{E}$ is a local Dirichlet form that possesses a squared field operator $\Gamma$ ([7], [17]).

We may define two operations on error structures that are compatible with the construction of probability spaces: the image of an error structure by an element of its domain ([7], p.186) and countable products ([7], p.203). By this way, error structures on the fundamental spaces encountered in stochastic models (Wiener space, Monte Carlo space, Poisson space) are easily obtained starting from elementary structures on $\mathbb{R}$ ([6], Chap.6).

Let us denote by $L^{2}([0,1])$, the space of the measurable functions on $[0,1]$ that are square integrable with respect to the Lebesgue measure. The following example describes one of the simplest error structure on the Wiener space that is intrinsically linked to the so-called Malliavin calculus ([18]). It provides an 
operational tool, easy to handle, to study the sensitivity of financial models to variations in the functional parameters ([6], Chap.7).

Example 1: Let $\mathcal{C}=\{f \in C([0,1], \mathbb{R}) \mid f(0)=0\}$ be the Wiener space equipped with the uniform norm $\|\cdot\|_{\infty}$ and $\mathcal{B}(\mathcal{C})$ the associated Borel $\sigma$-field. Let us denote by $\mu$ the Wiener measure on $\mathcal{C}$. Thus, with respect to $\mu$, the process $\left(B_{t}\right)_{t \in[0,1]}$ defined by $B_{t}: f \in \mathcal{C} \mapsto f(t)$ is a continuous Brownian motion. We consider the following subspace of $L^{2}(\mu)$

$\mathcal{A}=\left\{F\left(\int_{0}^{1} h_{1}(s) d B_{s}, . ., \int_{0}^{1} h_{n}(s) d B_{s}\right) ; n \in \mathbb{N}^{*}, F \in C^{1} \cap \operatorname{Lip}, h_{i} \in L^{2}([0,1])\right\}$.

It can be proved (cf [7], p.78) that there exists a unique error structure

$$
S_{O U}=\left(\mathcal{C}, \mathcal{B}(\mathcal{C}), \mu, \mathbb{D}_{O U}, \Gamma_{O U}\right)
$$

such that $\mathcal{A} \subset \mathbb{D}_{O U}\left(\mathbb{D}_{O U}\right.$ being minimal for inclusion) and $\forall h \in L^{2}([0,1])$

$$
\Gamma_{\text {OU }}\left[\int_{0}^{1} h(s) d B_{s}\right]=\int_{0}^{1} h^{2}(s) d s
$$

This structure is called the Ornstein-Uhlenbeck error structure on $\mathcal{C}$ •

As far as error calculus is concerned, it is important for a rational treatment to justify the choice of such a tool. In [4] Bouleau studies the polygonal approximation of Donsker on $[0,1]$

$$
X_{n}(t)=\frac{1}{\sqrt{n}}\left(\sum_{k=1}^{[n t]} U_{k}+(n t-[n t]) U_{[n t]+1}\right)
$$

when the random variables $\left(U_{k}\right)_{k \in \mathbb{N}^{*}}$ are supposed to be erroneous, the errors being modelised by error structures. He shows that under the assumptions of independence and stationarity on the errors, $X_{n}$ converges in the sense of Dirichlet forms (definition 2.0.5) toward $S_{O U}$. This study completes the results obtained in [8] concerning the central limit theorem in Hilbert spaces. For scalar parameters a general answer has been proposed in [5] in relation with the notion of Fisher's information.

The organization of the paper is as follows. In section 2 we present some preliminaries on the Dirichlet forms theory that will be used in the sequel. In section 3 we prove an extension of the preceding result providing the convergence in Dirichlet law of the process

$$
\int_{0}^{\cdot} h(s) d X_{n}(s), h \in L^{2}([0,1]) .
$$

The obtained result does not require regularity hypotheses on $h$. It is completed by the study of continuous-time approximations of some regular Gaussian processes including the fractional Brownian motion with Hurst parameter 
$H \in] 0,1[$. Finally, we are interested in section 4 in the refinement of a result of Bardina and Jolis ([2]) concerning the convergence of some multiple stochastic integrals

$$
\int_{[0, t]^{p}} h\left(x_{1}, \ldots, x_{p}\right) d X_{n}\left(x_{1}\right) \ldots d X_{n}\left(x_{p}\right)
$$

where $h$ is given by a multimeasure ([19]). In this case, thanks to a simple integration by parts, we show that (3) is given by a continuous operator of the process $X_{n}$ whose properties are compatible with error calculus.

The main tools used in this paper are the notion of the vectorial domain of a Dirichlet form (in the sense of Feyel and La Pradelle [11]), that allows an extension of the functional calculus (1) for a class of random variables with values in a Banach space, and the improvement (for the Wasserstein metric) of some purely probabilistic convergence theorems.

\section{Preliminaries}

In this section we recall briefly the basic definitions needed in the following. We refer the reader to [7], [12] and [17] for a detailed presentation.

One of the features of the operator $\Gamma$ is to be bilinear, thus, the computations may be awkward to perform. According to a result of Mokobovski ([7], p.242), it is often possible to overcome this problem introducing a linear operator related to $\Gamma$ that fulfills the classical chain rule. In this way, let us adopt the following notation: if $\left(E,\|.\|_{E}\right)$ is a normed vector space and $(W, \mathcal{W}, P)$ a probability space, $L^{2}(P ; E)$ will be the set of measurable functions $F: W \rightarrow E$ such that $\mathbb{E}_{P}\left[\|F\|_{E}^{2}\right]<\infty$.

Definition 2.0.1. We say that an error structure $S=(W, \mathcal{W}, P, \mathbb{D}, \Gamma)$ owns a gradient if the property $(G)$ is fulfilled:

There exists a separable Hilbert space $\left(\mathcal{H},\|\|_{\mathcal{H}}\right)$ and an operator $\nabla$ from $\mathbb{D}$ into $L^{2}(P ; \mathcal{H})$ called the gradient such that

$$
\forall U \in \mathbb{D},\|\nabla U\|_{\mathcal{H}}^{2}=\Gamma[U] .
$$

The notion of derivative is a slight variant of the preceding definition constructed with a copy $(\hat{W}, \hat{\mathcal{W}}, \hat{P})$ of $(W, \mathcal{W}, P)$. It was introduced by Feyel and La Pradelle in the Gaussian case and allows to define naturally a tensor product of $\mathbb{D}$ with a separable Banach space $B$ having a Schauder basis ([11], p.900 or [7], p.266).

Definition 2.0.2. Let $J$ be an isometry from $\mathcal{H}$ into $L^{2}(\hat{m})$. For $X \in \mathbb{D}$, we denote by $X^{\#}$ the derivative of $X$ defined by

$$
X^{\#}=J(\nabla X) \in L^{2}(m \times \hat{m}) .
$$


We can of course suppose (which we shall do) that $\forall h \in \mathcal{H}, \mathbb{E}_{\hat{P}}[J(h)]=0$.

Remark 1: In the framework of example 1, we can show that $S_{O U}$ owns a gradient denoted by $\nabla_{O U}$ (taking $\mathcal{H}=L^{2}([0,1])$ ) which is none other than the gradient in the Malliavin's sense (cf [18]) and that satisfies $\forall h \in L^{2}([0,1])$, $\nabla_{O U}\left[\int_{0}^{1} h(s) d B_{s}\right]=h$. Moreover, if we denote by $(\hat{\mathcal{C}}, \widehat{\mathcal{B}(\mathcal{C})}, \hat{\mu})$ a copy of $(\mathcal{C}, \mathcal{B}(\mathcal{C}), \mu)$ and $\left(\hat{B}_{t}\right)_{t \in[0,1]}$ the associated Brownian motion, we define a derivative operator on $\mathbb{D}_{O U}$ putting $\forall h \in L^{2}([0,1]), J(h)=\int_{0}^{1} h(s) d \hat{B}_{s}$. Let us remark that the derivative may be handled by Ito's calculus, a very useful feature when computing error propagation (cf [6], chap.7).

Let us denote by $B^{\prime}$ the topological dual space of $B$ and $<,>$ the duality between $B$ and $B^{\prime}$. We are now able to define the vectorial domain of $S$ fulfilling $(G)$.

Definition 2.0.3. Let us denote by $\mathbb{D}_{B}$ the vector space of random variables $U$ in $L^{2}(m ; B)$ such that there exists $g$ in $L^{2}(m \times \hat{m} ; B)$ so that

$$
\forall \lambda \in B^{\prime},\left\langle\lambda, U>\in \mathbb{D} \text { and }<\lambda, U>^{\#}=<\lambda, g>\right.\text {. }
$$

We then put $g=U^{\#}$ and one equips $\mathbb{D}_{B}$ with the norm

$$
\|U\|_{\mathbb{D}_{B}}=\left(\|U\|_{L^{2}(m ; B)}^{2}+\frac{1}{2}\left\|U^{\#}\right\|_{L^{2}(m \times \hat{m} ; B)}^{2}\right)^{\frac{1}{2}} .
$$

$\left(\right.$ Thus $\left.\mathbb{D}_{\mathbb{R}^{d}}=\mathbb{D}^{d}\right)$.

Several properties of the error structure $S$ may be extended to the elements of the vectorial domain. Thus, according to [8], p.7, it is possible to take the image of $S$ by a random variable $U$ in $\mathbb{D}_{B}$. This gives an error structure on $B$ :

Definition 2.0.4. Let $U \in \mathbb{D}_{B}$, the term $\left(B, \mathcal{B}(B), U_{*} P, C^{1}(B, \mathbb{R}) \cap \operatorname{Lip}, \Gamma_{U}\right)$ where $\forall F \in C^{1}(B, \mathbb{R}) \cap \operatorname{Lip}, \Gamma_{U}[F]=\mathbb{E}_{P}[\Gamma[F(U)] \mid U]$, is a closable error prestructure in the sense of $[6], p .44$. Let us denote by $U_{*} S$ its smallest closed extension, and by $\left(\mathcal{E}_{U}, \mathbb{D}_{U}\right)$ the associated Dirichlet form. The structure $U_{*} S$ is called the image of $S$ by $U$ and $\forall F \in \mathbb{D}_{U}$ we have $\mathcal{E}_{U}[F]=\mathcal{E}[F(U)]$.

Remark 2: When $h \in L^{2}([0,1])$, the continuous process $\int_{0} h(s) d B_{s}=$ $\left(\int_{0}^{t} h(s) d B_{s}\right)_{t \in[0,1]}$ belongs to $\left(\mathbb{D}_{O U}\right)_{\mathcal{C}}$. We denote by $S_{O U}^{h}$ the error structure $\left(\int_{0} h(s) d B_{s}\right)_{*} S_{O U}$ whose associated Dirichlet form $\mathcal{E}_{O U}^{h}$ satisfies $\forall F \in$ $C^{1}(\mathcal{C}, \mathbb{R}) \cap \operatorname{Lip}$

$$
\mathcal{E}_{O U}^{h}[F]=\int_{\mathcal{C}} \int_{\mathcal{C}}\left\langle F^{\prime}\left(\int_{0} h(s) d B_{s}\right), \int_{0}^{\cdot} h(s) d \hat{B}_{s}\right\rangle^{2} d \mu d \hat{\mu} . \bullet
$$


The following proposition ([8], p.5) extends the functional calculus.

Proposition 2.0.1. Let $F$ be a function from $B$ into $\mathbb{R}$ of class $C^{1}$ and Lipschitz. If $U \in \mathbb{D}_{B}, F(U) \in \mathbb{D}$ and

$$
F(U)^{\#}=<F^{\prime}(U), U^{\#}>
$$

thus

$$
\Gamma[F(U)]=\mathbb{E}_{\hat{P}}\left[<F^{\prime}(U), U^{\#}>^{2}\right] .
$$

Remark 3: We will see in section 4 (prop. 4.0.3) that the reinforcement of the integrability conditions on the pair $\left(U, U^{\#}\right)$ permits to generalize the preceding result to functions with polynomial growth.

Now, since the vectorial domain is stable by regular functions, we introduce a notion of convergence that extends to error structures the notion of weak convergence of random variables taking into account the underlying Dirichlet form.

Definition 2.0.5. We suppose that $S$ fulfills $(G)$. We say that a sequence $\left(U_{n}\right)_{n \in \mathbb{N}}$ in $\mathbb{D}_{B}$ converges in Dirichlet law if there exists an error structure $S_{0}=\left(B, \mathcal{B}(B), \nu, \mathbb{D}_{0}, \Gamma_{0}\right)$ such that :

i) $\left(U_{n}\right)_{*} P \underset{n \rightarrow \infty}{\longrightarrow} \nu$ weakly,

ii) $C^{1}(B, \mathbb{R}) \cap$ Lip $\subset \mathbb{D}_{0}$ and $\forall F \in C^{1}(B, \mathbb{R}) \cap \operatorname{Lip}, \mathcal{E}\left[F\left(U_{n}\right)\right] \underset{n \rightarrow \infty}{\longrightarrow} \mathcal{E}_{0}[F]$.

For convenience, we will say that $\left(U_{n}\right)_{n \in \mathbb{N}}$ converges in Dirichlet law toward $S_{0}$.

Remark 4: With the definition mentioned above the limit $S_{0}$ is in general not unique. To overcome this ambiguity, we have to impose to $\mathbb{D}_{0}$ to be minimal for inclusion. It is always possible because, by definition,

$$
\left(B, \mathcal{B}(B), \nu, C^{1}(B, \mathbb{R}) \cap \operatorname{Lip}, \Gamma_{0}\right)
$$

is a closable error pre-structure, thus, it possesses a smallest closed extension. Moreover, when $B=\mathbb{R}^{d}$, it is not necessary to suppose $(G)$ because the stability of $\mathbb{D}^{d}$ is given by (1). This hypothesis is the keystone of infinite dimensional studies.

\section{First results of convergence}

Throughout this section $K$ denotes a constant, independent of any parameter, whose value may change from case to case. Moreover, for any real valued function $h$, we will denote by $h_{+}$(resp. $h_{-}$) the positive (resp. negative) part of $h$. 
Let $\left(U_{k}\right)_{k \in \mathbb{N}^{*}}$ be a sequence of i.i.d random variables on the space $(W, \mathcal{W}, P)$. Assume further that the $U_{k}$ 's are square integrable, centered and normalized. Let $h \in L^{2}([0,1])$ and let $X_{n}$ be defined by $(2)$. We first study the convergence in Dirichlet law of the continuous stochastic process on $[0,1]$ defined as follows:

$$
Y_{n}^{h}(t)=\int_{0}^{t} h(s) d X_{n}(s)=\sqrt{n} \sum_{k=1}^{n} U_{k} \int_{\frac{k-1}{n}}^{\frac{k}{n}} h(s) I_{[0, t]}(s) d s .
$$

\subsection{Weak convergence of $Y_{n}^{h}$.}

We want to prove the weak convergence in $\mathcal{C}$ of $Y_{n}^{h}$ toward the corresponding Brownian integral. Since the process $X_{n}$ is a continuous semi-martingale, quiet general conditions, depending on the regularity of $h$, may be found in the literature. When $h$ is càd-làg (right continuous with left limit), a sufficient condition for $X_{n}$ is to satisfy the so-called U.T criterium proved by Jakubowski, Mémin and Pagès (cf [14] or [16] for an equivalent formulation). Since $X_{n}$ is a finite variation process, according to the proposition 6.12 p.378 of [13], the $U . T$ condition is equivalent to the tightness of the sequence $\left(\operatorname{Var}\left(X_{n}\right)_{t}\right)_{n \in \mathbb{N}^{*}}$, for all $t \in[0,1], \operatorname{Var}\left(X_{n}\right)$ being the variation process given by

$$
\operatorname{Var}\left(X_{n}\right)_{t}=\sup \sum\left|X\left(t_{k+1}\right)-X\left(t_{k}\right)\right|
$$

(where the supremum is over partitions of the interval $[0, t]$ ). But we have

$$
\operatorname{Var}\left(X_{n}\right)_{t}=\frac{1}{\sqrt{n}}\left(\sum_{k=1}^{[n t]}\left|U_{k}\right|+(n t-[n t])\left|U_{[n t]+1}\right|\right),
$$

hence this assumption is obviously not fulfilled. We can also find in [13] chap.9 another criterium that required the convergence for the distance in variation of the components of the characteristic of the semi-martingale $X_{n}$ toward those of the Brownian motion. Unfortunately it fails also in our framework. Thus, we are going to give a direct proof, supposing first that $h$ is continuous and concluding by approximations.

Proposition 3.1.1. When $h \in C([0,1], \mathbb{R})$, the sequence $\left(Y_{n}^{h}\right)$ weakly converges in $\mathcal{C}$ toward $Y^{h}=\int_{0} h(s) d B_{s}$.

Proof: We set, $\forall n \in \mathbb{N}^{*}, \forall k \in 0, \ldots, n, x_{k, n}=\frac{k}{n}$. Let us define

$$
\widetilde{Y_{n}^{h}}(t)=\frac{1}{\sqrt{n}}\left(\sum_{k=1}^{[n t]} h\left(x_{k, n}\right) U_{k}+(n t-[n t]) h\left(x_{[n t]+1, n}\right) U_{[n t]+1}\right) .
$$


According to a functional version of the Lindeberg-Feller's theorem ([10], p.226), $\widetilde{Y_{n}^{h}}$ weakly converges in $\mathcal{C}$ toward $Y^{h}$. To conclude it remains to show that the processes $Y_{n}^{h}$ and $\widetilde{Y_{n}^{h}}$ are contiguous meaning that, $\forall \varepsilon>0, P\left(\left\|\widetilde{Y_{n}^{h}}-Y_{n}^{h}\right\|_{\infty}>\right.$ $\varepsilon) \underset{n \rightarrow \infty}{\longrightarrow} 0$. But

$$
\begin{aligned}
Y_{n}^{h}(t)-\widetilde{Y_{n}^{h}}(t) & =\underbrace{\sqrt{n} \sum_{k=1}^{[n t]} U_{k} \int_{x_{k-1, n}}^{x_{k, n}}\left[h(s)-h\left(x_{k, n}\right)\right] d s}_{A_{n}(t)} \\
& +\underbrace{\sqrt{n} U_{[n t]+1} \int_{\frac{[n t]}{n}}^{t}\left[h(s)-h\left(x_{[n t]+1, n}\right)\right] d s}_{B_{n}(t)} .
\end{aligned}
$$

Since

$$
\left\|A_{n}\right\|_{\infty}=\sqrt{n} \operatorname{Max}_{1 \leq j \leq n}\left|\sum_{k=1}^{j} U_{k} \int_{x_{k-1, n}}^{x_{k, n}}\left[h(s)-h\left(x_{k, n}\right)\right] d s\right|,
$$

by Doob inequality

$$
\mathbb{E}\left[\left\|A_{n}\right\|_{\infty}^{2}\right] \leq 4 n \sum_{k=1}^{n}\left[\int_{x_{k-1, n}}^{x_{k, n}}\left[h(s)-h\left(x_{k, n}\right)\right] d s\right]^{2} .
$$

Using the uniform continuity of $h$, the right hand side of the preceding inequality tends to zero as $\mathrm{n}$ tends to infinity. For the other term we have

$$
\begin{aligned}
P\left(\left\|B_{n}\right\|_{\infty}>\varepsilon\right) & \leq P\left(\underset{1 \leq k \leq n}{\operatorname{Max}}\left|U_{k}\right|>\frac{\varepsilon \sqrt{n}}{2\|h\|_{\infty}}\right) \\
& \leq \sum_{k=1}^{n} P\left(\left|U_{k}\right|>\frac{\varepsilon \sqrt{n}}{2\|h\|_{\infty}}\right) \\
& \leq K \mathbb{E}\left[\left(U_{1}\right)^{2} I_{\left.\left\{\left|U_{1}\right|>\frac{\varepsilon \sqrt{n}}{2\|h\|_{\infty}}\right\}\right] \underset{n \rightarrow \infty}{\rightarrow} 0,} 0,\right.
\end{aligned}
$$

and the result follows.

The preceding argument does not remain valid for any $h \in L^{2}([0,1])$. In fact taking $h=I_{\mathbb{R} \backslash \mathbb{Q}}$ we see that $\widetilde{Y_{n}^{h}}=0$ and $Y_{n}^{h}=X_{n}$. Nevertheless the result still holds:

Proposition 3.1.2. When $h \in L^{2}([0,1])$, the sequence $\left(Y_{n}^{h}\right)$ converges weakly in $\mathcal{C}$ toward $Y^{h}$. 
Proof: According to the Portmanteau lemma [20] we only have to prove that for all $\Phi: \mathcal{C} \rightarrow \mathbb{R}$ Lipschitz and bounded

$$
\mathbb{E}_{P}\left[\Phi\left(Y_{n}^{h}\right)\right] \underset{n \rightarrow \infty}{\rightarrow} \mathbb{E}_{\mu}\left[\Phi\left(Y^{h}\right)\right]
$$

Let $g$ be a continuous function such that $\|h-g\|_{L^{2}([0,1])} \leq \varepsilon$. We have

$$
\mathbb{E}_{P}\left[\Phi\left(Y_{n}^{h}\right)\right]-\mathbb{E}_{\mu}\left[\Phi\left(Y^{h}\right)\right]=\alpha_{n}+\beta_{n}+\gamma_{n}
$$

where $\alpha_{n}=\mathbb{E}_{P}\left[\Phi\left(Y_{n}^{h}\right)-\Phi\left(Y_{n}^{g}\right)\right], \beta_{n}=\mathbb{E}_{P}\left[\Phi\left(Y_{n}^{g}\right)\right]-\mathbb{E}_{\mu}\left[\Phi\left(Y^{g}\right)\right]$ and $\gamma_{n}=$ $\mathbb{E}_{\mu}\left[\Phi\left(Y^{g}\right)-\Phi\left(Y^{h}\right)\right]$.

Since $\Phi$ is Lipschitz, Doob inequality implies

$$
\left|\gamma_{n}\right| \leq K \mathbb{E}_{\mu}\left[\left\|Y^{g}-Y^{h}\right\|_{\infty}^{2}\right]^{\frac{1}{2}} \leq 2 K\|h-g\|_{L^{2}([0,1])} \leq 2 K \varepsilon
$$

In the same way one has

$$
\alpha_{n} \leq K\left[\mathbb{E}_{P}\left[\left\|Y_{n}^{(g-h)_{+}}\right\|_{\infty}^{2}\right]^{\frac{1}{2}}+\mathbb{E}_{P}\left[\left\|Y_{n}^{(g-h)_{-}}\right\|_{\infty}^{2}\right]^{\frac{1}{2}}\right]=K\left[\alpha_{n, 1}+\alpha_{n, 2}\right] .
$$

The sample paths of the process $Y_{n}^{(g-h)_{+}}$being piecewise monotonic,

$$
\left|\alpha_{n, 1}\right| \leq K \mathbb{E}_{P}\left[\left\|Y_{n}^{(g-h)_{+}}\right\|_{\infty}^{2}\right]^{\frac{1}{2}}=K \mathbb{E}_{P}\left[n \operatorname{Max}_{1 \leq k \leq n}\left|\sum_{j=1}^{k} U_{j} \int_{\frac{j-1}{n}}^{\frac{j}{n}}(g-h)_{+}(s) d s\right|^{2}\right]^{\frac{1}{2}}
$$

By applying again the Doob inequality and the Schwarz inequality

$$
\left|\alpha_{n, 1}\right| \leq 2 K\left(n \sum_{j=1}^{n}\left[\int_{\frac{j-1}{n}}^{\frac{j}{n}}(g-h)_{+}(s) d s\right]^{2}\right)^{\frac{1}{2}} \leq 2 K \varepsilon .
$$

The same idea is used for $\alpha_{n, 2}$ and one obtains $\left|\alpha_{n, 2}\right| \leq 2 K \varepsilon$.

For $\beta_{n}$, we conclude using proposition 3.1.1 for the variable $Y_{n}^{g}$ because $\mathrm{g}$ is continuous.

Remark 5: Adapting the preceding proof, we can show that $Y_{n}^{h_{n}}$ converges weakly in $\mathcal{C}$ toward $Y^{h}$ when $\left\|h_{n}-h\right\|_{L^{2}([0,1])} \underset{n \rightarrow \infty}{\rightarrow} 0 . \bullet$

\subsection{Extension in the framework of Dirichlet forms.}

\subsubsection{Convergence of the finite dimensional distributions.}

Here we suppose that the $U_{k}$ 's are erroneous (errors being modelized by error structures) with a condition of independence and stationarity for the errors. In other words, the $U_{k}$ 's are the coordinate maps of the following product ([7], p.203)

$$
S=(W, \mathcal{W}, P, \mathbb{D}, \Gamma)=(\mathbb{R}, \mathcal{B}(\mathbb{R}), \lambda, d, \gamma)^{\mathbb{N}^{*}}
$$


the error structure $s=(\mathbb{R}, \mathcal{B}(\mathbb{R}), \lambda, d, \gamma)$ being such that the identity $i$ belongs to $d, \mathbb{E}_{\lambda}[i]=0$ and $\mathbb{E}_{\lambda}\left[i^{2}\right]=1$. We shall also assume without any loss of generality that $e[i]=1$ where $e$ is the Dirichlet form associated to $s$. Thus the $U_{k}$ 's are i.i.d random variables on $W$ with distribution $\lambda$ fulfilling $U_{k} \in \mathbb{D}$ and

$$
\Gamma\left[U_{n}, U_{m}\right]=\delta_{n}^{m} \gamma[i]\left(U_{n}\right)
$$

where $\delta_{n}^{m}=1$ if $n=m$ and $\delta_{n}^{m}=0$ otherwise. With these hypotheses $\left(U_{k}\right)_{k \in \mathbb{N}^{*}}$ is a sequence of Dirichlet independent random variables with the same Dirichlet law ([7] p.217).

First we prove a technical lemma that will be used hereafter.

Lemma 3.2.1. Suppose that $p$ is an even integer. When $h \in L^{p}([0,1])$ and $p \geq 1$ then

$$
V_{n}^{h, p}=n^{p-1} \sum_{j=1}^{n}\left[\int_{\frac{j-1}{n}}^{\frac{j}{n}} h(s) d s\right]_{n \rightarrow \infty}^{p} \int_{0}^{1} h^{p}(s) d s .
$$

Proof: This result is obvious when $h$ is continuous because, by the mean value theorem, $V_{n}^{h, p}$ is a Riemann type sum. For the general case we proceed by approximation. Let $g$ be a continuous function such that $\|h-g\|_{L^{p}([0,1])} \leq \varepsilon$. Then

$$
V_{n}^{h, p}-\int_{0}^{1} h^{p}(s) d s=\underbrace{V_{n}^{h, p}-V_{n}^{g, p}}_{\alpha_{n}}+\underbrace{V_{n}^{g, p}-\int_{0}^{1} g^{p}(s) d s}_{\beta_{n}}+\underbrace{\int_{0}^{1}\left(g^{p}-h^{p}\right)(s) d s}_{\gamma_{n}} .
$$

By Hölder inequality

$$
\operatorname{Max}\left(V_{n}^{h, p}, V_{n}^{g, p}\right) \leq \operatorname{Max}\left(\|h\|_{L^{p}([0,1])}^{p},\|g\|_{L^{p}([0,1])}^{p}\right) .
$$

Since $x \mapsto x^{p}$ is locally Lipschitz, Minkowski inequality gives $\left|\alpha_{n}\right| \leq K\left(V_{n}^{h-g, p}\right)^{\frac{1}{p}}$ $\leq K\|h-g\|_{L^{p}([0,1])} \leq K \varepsilon$ and $\left|\gamma_{n}\right| \leq K \varepsilon$. Finally, $g$ being continuous, the term $\beta_{n}$ converges to zero as $n$ goes to infinity.

Now, we are able to show the convergence in Dirichlet law of the finite dimensional distributions of $\left(Y_{n}^{h}\right)$.

Proposition 3.2.1. $\forall\left(t_{1}, \ldots, t_{p}\right) \in[0,1]^{p}$, the sequence $\left(Y_{n}^{h}\left(t_{1}\right), \ldots, Y_{n}^{h}\left(t_{p}\right)\right)$ converges in Dirichlet law toward $\left(Y^{h}\left(t_{1}\right), \ldots, Y^{h}\left(t_{p}\right)\right)_{*} S_{O U}$.

Proof: For lighten the notation let us suppose that $p=1$. The general case may be treated in the same way. By proposition 3.1.2 and definition 2.0.5, we only have to prove that for $F \in C^{1}(\mathbb{R}, \mathbb{R}) \cap$ Lip,

$$
2 \mathcal{E}\left[F\left(Y_{n}^{h}\left(t_{1}\right)\right)\right] \underset{n \rightarrow \infty}{\rightarrow} 2 \mathcal{E}_{O U}\left[F\left(Y^{h}\left(t_{1}\right)\right)\right]=\int_{0}^{t_{1}} h^{2}(s) d s \times \int_{\mathcal{C}} F^{\prime 2}\left(Y\left(t_{1}\right)\right) d \mu .
$$


From the functional calculus and the property (4) one has

$$
2 \mathcal{E}\left[F\left(Y_{n}\left(t_{1}\right)\right)\right]=\mathbb{E}_{P}\left[F^{\prime 2}\left(Y_{n}\left(t_{1}\right)\right) \Gamma\left[Y_{n}\left(t_{1}\right)\right]\right]
$$

with

$$
\Gamma\left[Y_{n}\left(t_{1}\right)\right]=n \sum_{k=1}^{\left[n t_{1}\right]} \gamma[i]\left(U_{k}\right)\left[\int_{\frac{k-1}{n}}^{\frac{k}{n}} h(s) d s\right]^{2}+\underbrace{n \gamma[i]\left(U_{\left[n t_{1}\right]+1}\right)\left[\int_{\frac{\left[n t_{1}\right]}{n}}^{t_{1}} h(s) d s\right]^{2}}_{Z_{n}\left(t_{1}\right)} .
$$

Since $\left|Z_{n}\left(t_{1}\right)\right| \leq \gamma[i]\left(U_{\left[n t_{1}\right]+1}\right) \int_{\frac{\left[n t_{1}\right]}{n}}^{t_{1}} h^{2}(s) d s, Z_{n}\left(t_{1}\right)$ goes to 0 in $L^{1}(P)$. Thus it remains to study the term

$$
n \mathbb{E}_{P}\left[F^{\prime 2}\left(Y_{n}\left(t_{1}\right)\right) \sum_{k=1}^{\left[n t_{1}\right]} \gamma[i]\left(U_{k}\right)\left[\int_{\frac{k-1}{n}}^{\frac{k}{n}} h(s) d s\right]^{2}\right]
$$

Let us denote by $\widehat{\psi_{n}}$ the Fourier transform of the measure $\nu_{n}$ defined by

$$
d \nu_{n}=Y_{n}\left(t_{1}\right)_{*}\left(n \sum_{k=1}^{\left[n t_{1}\right]} \gamma[i]\left(U_{k}\right)\left[\int_{\frac{k-1}{n}}^{\frac{k}{n}} h(s) d s\right]^{2} d P\right)
$$

The independence of the $\left(U_{k}\right)$ 's yields

$$
\widehat{\psi_{n}}(u)=\mathbb{E}_{P}\left[e^{i u Y_{n}\left(t_{1}\right)}\right]\left(n \sum_{k=1}^{\left[n t_{1}\right]}\left[\int_{\frac{k-1}{n}}^{\frac{k}{n}} h(s) d s\right]^{2} g_{n, k}(u)\right)
$$

where

$$
g_{n, k}(u)=\frac{\mathbb{E}_{P}\left[e^{i u \sqrt{n} U_{1} \int_{\frac{k-1}{n}}^{\frac{k}{n}} h(s) d s} \Gamma\left[U_{1}\right]\right]}{\mathbb{E}_{P}\left[e^{i u \sqrt{n} U_{1} \int_{\frac{k-1}{n}}^{\frac{k}{n}} h(s) d s}\right]} .
$$

But using the Cauchy-Schwarz inequality and the uniform continuity on $[0,1]$ of the function $\sqrt{\int_{0}^{\cdot} h^{2}(s) d s}, \underset{k \in\{0, \ldots, n\}}{\sup }\left|g_{n, k}(u)-1\right| \underset{n \rightarrow \infty}{\rightarrow} 0$. Moreover,

$n \sum_{k=1}^{n}\left[\int_{\frac{k-1}{n}}^{\frac{k}{n}} I_{\left[0, t_{1}\right]}(s) h(s) d s\right]^{2}-n \sum_{k=1}^{\left[n t_{1}\right]}\left[\int_{\frac{k-1}{n}}^{\frac{k}{n}} h(s) d s\right]^{2}=n\left[\int_{\frac{\left[n t_{1}\right]}{n}}^{t_{1}} h(s) d s\right]_{n \rightarrow \infty}^{2} 0$.

To complete the proof it suffices to use the lemma 3.2.1 and the proposition 3.1.2. $\square$ 


\subsubsection{Functional convergence.}

In order to propose a functional extension of the preceding proposition we are going to assume that the error structure $s=(\mathbb{R}, \mathcal{B}(\mathbb{R}), \lambda, d, \gamma)$ fulfills the property $(G)$ and we denote by ' an associated derivative operator built with a copy $(\widehat{\mathbb{R}}, \widehat{\mathcal{B}(\mathbb{R})}, \widehat{\lambda})$ of $(\mathbb{R}, \mathcal{B}(\mathbb{R}), \lambda)$ (the choice of the isometry, although noncanonical, is not specified). Let \# be the derivative operator on the product structure $S=(W, \mathcal{W}, P, \mathbb{D}, \Gamma)$ that can be deduced from ' (cf [6] p.79). If the $\widehat{U_{k}}$ 's are the coordinate maps of $(\widehat{W}, \widehat{\mathcal{W}}, \widehat{P})$, one has, for $p \in \mathbb{N}^{*}$ and $F \in C^{1}\left(\mathbb{R}^{p}, \mathbb{R}\right) \cap$ Lip,

$$
F\left(U_{1}, \ldots, U_{p}\right)^{\#}=\sum_{k=1}^{p} F_{k}^{\prime}\left(U_{1}, \ldots, U_{p}\right) i^{\prime}\left(U_{k}, \widehat{U_{k}}\right) .
$$

Hence, we show easily that $Y_{n}$ belongs to $\mathbb{D}_{\mathcal{C}}$ and that

$$
\left(Y_{n}^{h}\right)^{\#}(t)=\sqrt{n} \sum_{k=1}^{n} U_{k}^{\#} \int_{\frac{k-1}{n}}^{\frac{k}{n}} h(s) I_{[0, t]}(s) d s .
$$

According to the extended functional calculus (proposition 2.0.1), if $F \in$ $C^{1}(\mathcal{C}, \mathbb{R}) \cap \operatorname{Lip}$,

$$
\mathcal{E}\left[F\left(Y_{n}^{h}\right)\right]=\int_{W} \int_{\widehat{W}}<F^{\prime}\left(Y_{n}^{h}\right),\left(Y_{n}^{h}\right)^{\#}>^{2} d \widehat{P} d P .
$$

The pairs $\left(U_{k}, \widehat{U_{k}}\right)$ being $i . i . d$, the extension of the proposition 3.1.2 for vector valued random variables (that is obvious) ensures the weak convergence in $\mathcal{C}^{2}$ of $\left(Y_{n}^{h},\left(Y_{n}^{h}\right)^{\#}\right)$ toward $\left(\int_{0} h(s) d B_{s}, \int_{0} h(s) d \widehat{B}_{s}\right)$. Since $\phi:(x, y) \in \mathcal{C}^{2} \mapsto<$ $F^{\prime}(x), y>^{2}$ is a continuous function with quadratic growth, the conclusion holds if we are able to prove the following extension of the proposition 3.1.2 (that was proved for $h=1$ in [4]).

Proposition 3.2.2. Let $\phi: \mathcal{C} \rightarrow \mathbb{R}$ a continuous function such that $\forall x \in \mathcal{C}$, $|\phi(x)| \leq K\left(1+\|x\|_{\infty}^{2}\right)$,

$$
\mathbb{E}_{P}\left[\phi\left(Y_{n}^{h}\right)\right] \underset{n \rightarrow \infty}{\rightarrow} \mathbb{E}_{\mu}\left[\phi\left(Y^{h}\right)\right]
$$

The following lemma will be the cornerstone of the proof (cf [3] p.69).

Lemma 3.2.2. Let $\left(\xi_{1}, \ldots, \xi_{n}\right)$ be independent random variables with variances $\left(\sigma_{1}^{2}, \ldots, \sigma_{n}^{2}\right)$. Denote $\forall 1 \leq i \leq n, s_{i}^{2}=\sigma_{1}^{2}+\ldots+\sigma_{i}^{2}$ and $S_{i}=\xi_{1}+\ldots+\xi_{i}$, thus $\forall \lambda \in \mathbb{R}$,

$$
P\left(\underset{1 \leq i \leq n}{\operatorname{Max}}\left|S_{i}\right| \geq \lambda s_{n}\right) \leq 2 P\left(\left|S_{n}\right| \geq(\lambda-\sqrt{2}) s_{n}\right) .
$$


Proof of the proposition 3.2.2: Classically, we only have to show the uniform integrability of the $\left\|Y_{n}^{h}\right\|_{\infty}^{2}$ 's. Let $\alpha \in \mathbb{R}_{+}$, we are interested in the following quantity

$$
A_{n, \alpha}=\mathbb{E}_{P}\left[\left\|Y_{n}^{h}\right\|_{\infty}^{2} I_{\left\{\left\|Y_{n}^{h}\right\|_{\infty}^{2} \geq \alpha\right\}}\right]
$$

By Fubini theorem,

$$
A_{n, \alpha}=\alpha P\left(\left\|Y_{n}^{h}\right\|_{\infty}^{2} \geq \alpha\right)+\int_{\alpha}^{\infty} P\left(\left\|Y_{n}^{h}\right\|_{\infty}^{2} \geq t\right) d t
$$

We set $Y_{n}^{h_{+}}(t)=\int_{0}^{t} h_{+}(s) d X_{n}(s)$ and $Y_{n}^{h_{-}}(t)=\int_{0}^{t} h_{-}(s) d X_{n}(s)$, thus

$$
\begin{aligned}
A_{n, \alpha} & \leq \underbrace{\alpha P\left(\left\|Y_{n}^{h_{+}}\right\|_{\infty}^{2} \geq \frac{\alpha}{4}\right)+\int_{\alpha}^{\infty} P\left(\left\|Y_{n}^{h_{+}}\right\|_{B}^{2} \geq \frac{t}{4}\right) d t}_{B_{n, \alpha}} \\
+ & \underbrace{\alpha P\left(\left\|Y_{n}^{h_{-}}\right\|_{\infty}^{2} \geq \frac{\alpha}{4}\right)+\int_{\alpha}^{\infty} P\left(\left\|Y_{n}^{h_{-}}\right\|_{B}^{2} \geq \frac{t}{4}\right) d t}_{C_{n, \alpha}} .
\end{aligned}
$$

We are going to show that $\lim \sup B_{n, \alpha} \underset{\alpha \rightarrow \infty}{\rightarrow} 0$, the method being similar for the term $C_{n, \alpha}$. Since $h_{+} \geq 0$, the sample paths of the process $Y_{n}^{h_{+}}$are piecewise monotonic thus

$$
\left\|Y_{n}^{h_{+}}\right\|_{\infty}=\sqrt{n} \operatorname{Max}_{1 \leq k \leq n}\left|\sum_{j=1}^{k} U_{j} \int_{\frac{j-1}{n}}^{\frac{j}{n}} h_{+}(s) d s\right| .
$$

By lemma 3.2.2 one has

$$
P\left(\left\|Y_{n}^{h_{+}}\right\|_{\infty} \geq t\right) \leq 2 P\left(\left|\sqrt{n} \sum_{k=1}^{n} U_{j} \int_{\frac{k-1}{n}}^{\frac{k}{n}} h_{+}(s) d s\right| \geq t-\sqrt{2 s_{n}^{2}}\right)
$$

where $s_{n}^{2}=n \sum_{k=1}^{n}\left[\int_{\frac{k-1}{n}}^{\frac{k}{n}} h_{+}(s) d s\right]^{2}$. Hence,

$$
B_{n, \alpha} \leq 2 \alpha P\left(\left|Y_{n}^{h_{+}}(1)\right| \geq \frac{\sqrt{\alpha}}{2}-\sqrt{2 s_{n}^{2}}\right)+2 \mathbb{E}_{P}\left[\left(\left[Y_{n}^{h_{+}}(1)+\sqrt{2 s_{n}^{2}}\right]^{2}-\alpha\right)_{+}\right] \text {. }
$$

It comes now from the proposition 3.1.2 that $\frac{\left|Y_{n}^{h_{+}}(1)\right|}{\sqrt{\int_{0}^{1} h_{+}^{2}(s) d(s)}}$ converges in distribution toward $|N|$ where $N$ is a centered and reduced normal variable. Moreover by lemma 3.2.1, $\sqrt{2 s_{n}^{2}} \underset{n \rightarrow \infty}{\rightarrow} \sqrt{2 \int_{0}^{1} h_{+}^{2}(s) d(s)}$. Using Dini theorem

$$
\alpha P\left(\left|Y_{n}^{h_{+}}(1)\right| \geq \frac{\sqrt{\alpha}}{2}-\sqrt{2 s_{n}^{2}}\right) \underset{n \rightarrow \infty}{\rightarrow} \alpha P\left(|N| \geq \frac{\sqrt{\alpha}}{2 \sqrt{\int_{0}^{1} h_{+}^{2}(s) d(s)}}-\sqrt{2}\right) .
$$


1018

Finally, by independence of the $U_{k}$ 's, the random variables $\left(Y_{n}^{h_{+}}(1)\right)^{2}$ are uniformly integrable, it follows that

$$
\mathbb{E}_{P}\left[\left(\left[Y_{n}^{h_{+}}(1)+\sqrt{2 s_{n}^{2}}\right]^{2}-\alpha\right)_{+}\right] \underset{n \rightarrow \infty}{\rightarrow} \mathbb{E}_{P}\left[\left(\int_{0}^{1} h_{+}^{2}(s) d s[N+\sqrt{2}]^{2}-\alpha\right)_{+}\right]
$$

and that $\lim _{n} \sup B_{n, \alpha} \underset{\alpha \rightarrow \infty}{\rightarrow} 0 . \square$

Applying the preceding result to (5) gives

$$
\mathcal{E}\left[F\left(Y_{n}^{h}\right)\right] \underset{n \rightarrow \infty}{\rightarrow} \int_{\mathcal{C}} \int_{\mathcal{C}}\left\langle F^{\prime}\left(\int_{0} h(s) d B_{s}\right), \int_{0}^{\cdot} h(s) d \hat{B}_{s}\right\rangle^{2} d \mu d \hat{\mu}
$$

thus according to remark 2 we have the expected result:

Proposition 3.2.3. The sequence $\left(Y_{n}^{h}\right)$ converges in Dirichlet law toward $S_{O U}^{h}$.

Remark 6: Taking $h=1$ we rediscover the extension of the Donsker's invariance principle obtained in [4].

\subsection{The case of general Gaussian processes.}

We work in the framework of section 3.2.2. Let $\left(Y^{K}(t)\right)_{t \in[0,1]}$ be a continuous Gaussian process of the form $Y^{K}(t)=\int_{0}^{1} K(t, s) d B_{s}$. Suppose that the kernel $K:[0,1]^{2} \rightarrow \mathbb{R}$ fulfills the two following properties :

i) $\mathrm{K}$ is measurable and $K(0, r)=0, r \in[0,1]$.

ii) There exists a continuous increasing function $G:[0,1] \rightarrow \mathbb{R}$ and a positive constant $\alpha$ such that for all $0 \leq t_{1}<t_{2} \leq 1$

$$
\int_{0}^{1}\left(K\left(t_{2}, r\right)-K\left(t_{1}, r\right)\right)^{2} d r \leq\left(G\left(t_{2}\right)-G\left(t_{1}\right)\right)^{\alpha} .
$$

Remark 7: We can find in [9] several examples of processes satisfying the preceding conditions. This is the case of the fractional Brownian motion with Hurst parameter $0<H<1$ and of the Ornstein-Uhlenbeck process.

Here we are interested in the convergence in Dirichlet law of the process $Y_{n}^{K}=\int_{0}^{1} K(., s) d X_{n}(s)$. First, we have the following lemma easily derived from definition 2.0.3.

Lemma 3.3.1. The process $Y_{n}^{K}\left(\right.$ resp. $\left.Y^{K}\right)$ belongs to $\mathbb{D}_{\mathcal{C}}\left(\right.$ resp. $\left.\left(\mathbb{D}_{O U}\right)_{\mathcal{C}}\right)$ with

$$
\left(Y_{n}^{K}\right)^{\#}=\int_{0}^{1} K(., s) d X_{n}^{\#}(s)\left(\operatorname{resp} .\left(Y^{K}\right)^{\#}=\int_{0}^{1} K(., s) d \widehat{B}_{s}\right) .
$$


Remark 8: By the preceding lemma we can consider the error structure $\left(Y^{K}\right)_{*} S_{O U}$ on the Wiener space. This structure that fulfills $(G)$ has been studied in [1] where the authors develop a stochastic calculus with respect to $Y^{K}$ and describe its properties according to the regularity of $K$.

When $K(t, s)=I_{[0, t]}(s) h(s)$ with $h \in L^{2}([0,1])$, one has $Y_{n}^{K}=Y_{n}^{h}$. Unfortunately, the sample paths of $Y_{n}^{K}$ are no more piecewise monotonic (it was the keystone in the proofs of propositions 3.1.2 and 3.2.2). Nevertheless imposing stronger integrability conditions on the $U_{k}$ 's we obtain the following result

Proposition 3.3.1. Suppose that $U_{1} \in L^{p}(P)$ with $p>\frac{2}{\alpha} \vee 2$. Then the process $Y_{n}^{K}$ converges weakly in $\mathcal{C}$ toward $Y^{K}$ and the random variables $\left\|Y_{n}^{K}\right\|_{\infty}^{2}$ are uniformly integrable.

Preuve: For the weak convergence, we recall that (cf. [2]) when $U_{1} \in L^{p}(P)$, $f \in L^{2}([0,1])$, there exists a constant $C_{p}$ independent of $n$ such that

$$
\mathbb{E}_{P}\left[\left(\int_{0}^{1} f(s) d X_{n}(s)\right)^{p}\right] \leq C_{p}\left(\int_{0}^{1} f^{2}(s) d s\right)^{\frac{p}{2}}
$$

We conclude using the same argument than in the proof of theorem 1 of [9].

To show the uniform integrability of the random variables $\left\|Y_{n}^{K}\right\|_{\infty}^{2}$ it suffices to prove the existence of a constant $\beta>2$ such that $\sup _{n \in \mathbb{N}^{*}} \mathbb{E}_{P}\left[\left\|Y_{n}^{K}\right\|^{\beta}\right]<\infty$. Using the hypothesis $i i$ ) and the inequality (6) one has $\forall 0 \leq t_{1}<t_{2} \leq 1$,

$$
\mathbb{E}_{P}\left[\left(Y_{n}^{K}\left(t_{1}\right)-Y_{n}^{K}\left(t_{2}\right)\right)^{p}\right] \leq C_{p}\left(G\left(t_{1}\right)-G\left(t_{2}\right)\right)^{\frac{p \alpha}{2}} .
$$

According to [3], p.95, the preceding inequality yields $\forall \delta>0, \exists C_{\delta}>0, \forall \varepsilon>0$,

$$
F_{n, \delta}(\varepsilon)=P\left(\sup _{|s-t| \leq \delta}\left|Y_{n}^{K}(t)-Y_{n}^{K}(s)\right| \geq \varepsilon\right) \leq \frac{C_{\delta}}{\varepsilon^{p}}
$$

Let $\varepsilon_{0}>0$ be such that $\beta=p-\varepsilon_{0}>2$, by (8) and an integration by parts

$$
\mathbb{E}_{P}\left[\sup _{|s-t| \leq \delta}\left|Y_{n}^{K}(t)-Y_{n}^{K}(s)\right|^{\beta}\right] \leq \beta\left(\int_{0}^{\infty} y^{\beta-1} F_{n, \delta}(y) d y\right) \leq \beta\left(1+\frac{C_{\delta}}{\varepsilon_{0}}\right)
$$

Since

$$
\mathbb{E}_{P}\left[\left\|Y_{n}^{K}\right\|^{\beta}\right] \leq 2^{\beta}\left(\mathbb{E}_{P}\left[\sup _{|s-t| \leq \frac{1}{2}}\left|Y_{n}^{K}(t)-Y_{n}^{K}(s)\right|^{\beta}\right]+\mathbb{E}_{P}\left[\left|Y_{n}^{K}(1 / 2)\right|^{\beta}\right]\right),
$$

the result comes from (7) and (9).

Using the same reasoning than in section 3.2.2 we have the following result: 
1020

Proposition 3.3.2. If the kernel $K$ fulfills properties $i)$ and $i i)$ and if $\left(U_{1}, U_{1}^{\#}\right) \in$ $L^{p}(W) \times L^{p}(W \otimes \hat{W})$ with $p>\frac{2}{\alpha} \vee 2$ then the process $Y_{n}^{K}$ converges in Dirichlet law toward $\left(Y^{K}\right)_{*} S_{O U}$.

\section{Convergence of multiple integrals given by a multi-measure}

Example 2: When the function $h$ is sufficiently regular, the proposition 3.2.3 is a direct consequence of the convergence in Dirichlet law of $X_{n}$ toward $S_{O U}$ that was proved in [4]. In fact, assume that $h$ is a right continuous function with finite variation and let us denote by $\nu$ the signed radon measure such that $\nu([t, 1])=h(t)$. For all $t \in[0,1]$, we define the measure $\bar{\nu}_{t}$ fulfilling $\forall A \in \mathcal{B}([0,1]), \bar{\nu}_{t}(A)=\nu(A(t))$ where $A(t)=A \cap[0, t]$ if $t \notin A$ and $A(t)=$ $A \cup[t, 1]$ if $t \in A$. An integration by parts yields

$$
\int_{0}^{t} h(s) d X_{n}(s)=\int_{0}^{t} X_{n}(s) d \bar{\nu}_{t}(s)=\phi_{h}\left(X_{n}\right)
$$

where $\phi_{h}: \mathcal{C} \rightarrow \mathcal{C}$ is of class $C^{1}$ and Lipschitz. Hence, since $X_{n}$ converges in Dirichlet law, $\phi_{h}\left(X_{n}\right)$ converges in Dirichlet law toward $\phi_{h}{ }_{*} S_{O U}$. But it comes from the Ito Formula that

$$
\int_{0}^{t} h(s) d B_{s}=\int_{0}^{t} B_{s} d \bar{\nu}_{t}(s)
$$

thus

$$
\phi_{h * S_{O U}}=\left(\int_{0}^{\cdot} h(s) d B_{s}\right) * S_{O U} \bullet
$$

Following example 2, we study the convergence of multiple integrals with regular integrands. Let $h: \mathbb{R}^{p} \rightarrow \mathbb{R}$ be a symmetric function, we are interested in the convergence in Dirichlet law of

$$
\int_{[0, t]^{p}} h\left(x_{1}, \ldots, x_{p}\right) d X_{n}\left(x_{1}\right) \ldots d X_{n}\left(x_{p}\right) .
$$

The weak convergence of (11) was proved in [2] studying the question of the continuous extension on the Wiener space of the functional

$$
\phi_{h}: \eta \in \mathcal{H} \mapsto \int_{[0, .]^{p}} h\left(x_{1}, \ldots, x_{p}\right) d \eta\left(x_{1}\right) \ldots d \eta\left(x_{p}\right) \in \mathcal{C}
$$

$\mathcal{H}$ being the Cameron-Martin space. In this way, we need the notion of a multimeasure (in the sense of Nualart and Zakai [19]) that extends to functions of several variables the notion of finite variation functions. 
Definition 4.0.1. A mapping $\nu:(\mathcal{B}([0,1]))^{p} \rightarrow \mathbb{R}$ is said to be a multimeasure if $\forall i \in\{1, \ldots, p\}, \forall\left(A_{1}, \ldots, A_{i-1}, A_{i+1}, \ldots, A_{p}\right) \in(\mathcal{B}([0,1]))^{p-1}$, the function

$$
A \in \mathcal{B}([0,1]) \mapsto \nu\left(A_{1}, \ldots, A_{i-1}, A, A_{i+1}, \ldots, A_{p}\right)
$$

is a signed measure. Moreover we say that $h$ is given by a multimeasure $\nu$ if

$$
h\left(x_{1}, \ldots, x_{p}\right)=\nu\left(\left[x_{1}, 1\right], \ldots,\left[x_{p}, 1\right]\right) .
$$

Thus, the answer is the following ([2], p.281):

Theorem 4.0.1. The following statement are equivalent:

a) $\phi_{h}$ possesses a continuous extension on $\mathcal{C}$.

b) The function $h$ is given by a multimeasure $\nu$.

Moreover, the extension of $\phi_{h}$ is such that $\forall \eta \in \mathcal{C}$

$$
\phi_{h}(\eta)=\int_{[0,]^{p}} \eta\left(x_{1}\right) \ldots \eta\left(x_{p}\right) d \bar{\nu}_{.}\left(x_{1}, \ldots, x_{n}\right)
$$

where $\left(\bar{\nu}_{t}\right)_{t \in[0,1]}$ is a family of multimeasure that satisfies

$$
\left\|\bar{\nu}_{t}\right\|_{F V} \leq\|\nu\|_{F V}\left(\|\|_{F V} \text { being the Frechet variation. }\right)
$$

Thus in the framework of the preceding theorem $\phi_{h}$ is continuous on $\mathcal{C}$. Since $X_{n}$ converges weakly in $\mathcal{C}$ toward the Brownian motion, by the continuous mapping theorem, $\phi_{h}\left(X_{n}\right)$ converges weakly in $\mathcal{C}$ toward $\phi_{h}(B$.$) which is none$ other than the multiple Stratonovich integral of $h$. Nevertheless, to deduce easily the convergence in Dirichlet law of $\phi_{h}\left(X_{n}\right)$ from $X_{n}$ 's one, we should have that $\phi_{h}$ belongs to $C^{1}(B, \mathbb{R}) \cap$ Lip. This is not the case in general (except for $p=1), \phi_{h}$ is only of class $C^{1}$ and fulfills

$$
\begin{gathered}
\left|\phi_{h}(x)\right| \leq\|\nu\|_{F V}\|x\|_{\infty}^{p}, \\
\left|\phi_{h}^{\prime}(x)[\tilde{x}]\right| \leq p\|\nu\|_{F V}\|\tilde{x}\|_{\infty}\|x\|_{\infty}^{p-1} .
\end{gathered}
$$

We overcome this problem imposing some integrability conditions on the pair $\left(U_{k}, U_{k}^{\#}\right)$.

First, we state the following technical lemma that extends in some sense [7] p.39.

Proposition 4.0.3. Let $S=(W, \mathcal{W}, P, \mathbb{D}, \Gamma)$ be an error structure that owns a gradient and $B$ a Banach space having a Schauder basis. Let $X \in \mathbb{D}_{B}$ such that $\|X\|_{B} \in L^{2 p}(W)$ and $\left\|X^{\#}\right\|_{B} \in L^{2 p}(W \otimes \hat{W})$. If $F \in C^{1}(B, \mathbb{R})$ fulfills $|F(x)| \leq K\|x\|_{B}^{p}$ and $\left\|F^{\prime}(x)\right\| \leq K\|x\|_{B}^{p-1}$, then

$$
F(X) \in \mathbb{D} \text { and } F(X)^{\#}=F^{\prime}(X)\left[X^{\#}\right] .
$$


Proof: Let us first suppose that $F$ is of the form $f\left(\lambda_{1}, \ldots, \lambda_{q}\right)$ with $\forall i \in$ $\{1, \ldots, q\}, \lambda_{i} \in B^{\prime}$ and $f \in C^{1}\left(\mathbb{R}^{q}, \mathbb{R}\right)$. Let $\left(\psi_{m}\right)_{m \geq 0}$ be a sequence of functions in $C^{1}(\mathbb{R}, \mathbb{R})$ with compact support such that

$$
\begin{aligned}
& \forall x, \lim _{m \rightarrow \infty} \psi_{m}(x)=x \text { and } \lim _{m \rightarrow \infty} \psi_{m}^{\prime}(x)=1, \\
& \forall m, \forall x,\left|\psi_{m}(x)\right| \leq|x| \text { and }\left|\psi_{m}^{\prime}(x)\right| \leq 1 .
\end{aligned}
$$

Denote $\Psi_{m}\left(x_{1}, . ., x_{q}\right)=f\left(\psi_{m}\left(x_{1}\right), \ldots, \psi_{m}\left(x_{q}\right)\right)$. By dominated convergence

$$
\Psi_{m}\left(\lambda_{1}(X), . ., \lambda_{q}(X)\right) \underset{m \rightarrow \infty}{\rightarrow} F(X) \text { in } L^{2}(W) .
$$

Since $\Psi_{m} \in C^{1}\left(\mathbb{R}^{q}, \mathbb{R}\right) \cap$ Lip and $X \in \mathbb{D}_{B}$ one has

$$
\Psi_{m}\left(\lambda_{1}(X), . ., \lambda_{q}(X)\right)^{\#}=\sum_{i=1}^{q} f_{i}^{\prime}\left(\psi_{m}\left(\lambda_{1}(X)\right), \ldots, \psi_{m}\left(\lambda_{q}(X)\right)\right) \psi_{m}^{\prime}\left(\lambda_{i}(X)\right) \lambda_{i}\left(X^{\#}\right)
$$

and dominated convergence implies

$$
\Psi_{m}\left(\lambda_{1}(X), . ., \lambda_{q}(X)\right)^{\#} \underset{m \rightarrow \infty}{\rightarrow} F^{\prime}(X)\left[X^{\#}\right] \text { in } L^{2}(W \otimes \hat{W}) .
$$

In the same way we show that

$$
\begin{gathered}
\Psi_{m}\left(\lambda_{1}(X), . ., \lambda_{q}(X)\right)-\Psi_{\tilde{m}}\left(\lambda_{1}(X), . ., \lambda_{q}(X)\right) \underset{m, \tilde{m} \rightarrow \infty}{\rightarrow} 0 \text { in } L^{2}(W), \\
\Psi_{m}\left(\lambda_{1}(X), . ., \lambda_{q}(X)\right)^{\#}-\Psi_{\tilde{m}}\left(\lambda_{1}(X), . ., \lambda_{q}(X)\right)^{\#} \underset{m, \tilde{m} \rightarrow \infty}{\rightarrow} 0 \text { in } L^{2}(W \otimes \hat{W}) .
\end{gathered}
$$

The result follows using the closedness of the operator \#.

For the general case, since $B$ is a Banach space having a Schauder basis, $F$ has to be approximated by cylinder functions. $\square$

Remark 9: When $B=\mathbb{R}$ the integrability conditions on $X$ and $X^{\#}$ involve to $X \in \mathbb{D}_{2 p}$ (in the sense of [7], p.39). Thus, proposition 4.0.3 is a consequence of [7], propo.6.2.2, p.39 when $B$ is finite dimensional. Nevertheless the conditions of [7] can't be transposed in the infinite dimensional setting because the operator $\Gamma$ can't be extended to $\mathbb{D}_{B}$ contrary to the derivative.

We shall deduce from the preceding proposition a functional calculus fulfilled by $X_{n}$.

Proposition 4.0.4. Suppose that $\left(U_{k}, U_{k}^{\#}\right) \in L^{2 p}(W) \times L^{2 p}(W \otimes \hat{W})$ then $\forall F \in C^{1}(\mathcal{C}, \mathbb{R})$ such that $|F(x)| \leq K\|x\|_{\infty}^{p}$ and $\left\|F^{\prime}(x)\right\| \leq K\|x\|_{\infty}^{p-1}$,

$$
F\left(X_{n}\right) \in \mathbb{D} \text { and } F\left(X_{n}\right)^{\#}=F^{\prime}\left(X_{n}\right)\left[X_{n}^{\#}\right] \text {. }
$$

Proof: Since $U_{k} \in L^{2 p}$ and $\left\|X_{n}\right\|_{\infty}=\max _{1 \leq k \leq n}\left|\frac{1}{\sqrt{n}} \sum_{j=1}^{k} U_{j}\right|$, it follows from Doob inequality that $\left\|X_{n}\right\|_{\infty} \in L^{2 p}(W)\left(\right.$ similarly $\left.\left\|X_{n}^{\#}\right\|_{\infty}^{p} \in L^{2}(W \otimes \hat{W})\right)$. 
Corollary 4.0.1. a) If $\left(U_{k}, U_{k}^{\#}\right) \in L^{2 p}(W) \times L^{2 p}(W \otimes \hat{W}), \phi_{h}\left(X_{n}\right) \in \mathbb{D}_{\mathcal{C}}$ and $\phi_{h}\left(X_{n}\right)^{\#}=\phi_{h}^{\prime}\left(X_{n}\right)\left[X_{n}^{\#}\right]$.

b) $\phi_{h}(B) \in\left(\mathbb{D}_{O U}\right)_{\mathcal{C}}$ and $\phi_{h}(B)^{\#}=\phi_{h}^{\prime}(B)[\hat{B}]$.

Proof: $a$ ) is obvious using proposition 4.0.4 and $b$ ) comes from proposition 4.0.3 by the properties of the Brownian motion.

Now we are able to study the convergence in Dirichlet law of $\phi_{h}\left(X_{n}\right)$ toward $\phi_{h}{ }_{*} S_{O U}$. Let $F \in C^{1}(B, \mathbb{R}) \cap \operatorname{Lip}$. We put $G=F\left(\phi_{h}\right)$, by $(13)$ and (14) and corollary 4.0.1,

$$
\mathcal{E}\left[F\left(\phi_{h}\left(X_{n}\right)\right)\right]=\int_{W} \int_{\hat{W}}\left(G^{\prime}\left(X_{n}\right)\left[X_{n}^{\#}\right]\right)^{2} d P d \hat{P}=\int_{W} \int_{\hat{W}} \Phi\left(X_{n}, X_{n}^{\#}\right) d P d \hat{P}
$$

where $\Phi: \mathcal{C}^{2} \rightarrow \mathbb{R}$ is a continuous function such that

$$
|\Phi(x, y)| \leq K\|x\|_{\infty}^{2(p-1)}\|y\|_{\infty}^{2} \leq K \max \left(\|x\|_{\infty},\|y\|_{\infty}\right)^{2 p} .
$$

We concluded using the following extension of the Donsker's theorem that has been proved for the case $q=2$ in [4].

Proposition 4.0.5. Let $\Phi: \mathcal{C} \rightarrow \mathbb{R}$ be a continuous function such that $\forall x \in \mathcal{C}$, $|\Phi(x)| \leq K\left(1+\|x\|_{\infty}^{q}\right)$. When $U_{1} \in L^{q}(W)$

$$
\mathbb{E}_{P}\left[\Phi\left(X_{n}\right)\right] \underset{n \rightarrow \infty}{\rightarrow} \mathbb{E}_{\mu}[\Phi]
$$

Proof: We just have to show the uniform integrability of $\left\|X_{n}\right\|_{\infty}^{q}$. The same argument than in the proof of proposition 3.2.2 yields for large $\alpha$

$A_{n, \alpha}=\mathbb{E}_{P}\left[\left\|X_{n}\right\|_{\infty}^{q} I_{\left\{\left\|X_{n}\right\|_{\infty}^{q} \geq \alpha\right\}}\right] \leq 2 \alpha P\left(\frac{\left|S_{n}\right|}{\sqrt{n}} \geq \frac{\alpha^{\frac{1}{q}}}{2}\right)+2 \mathbb{E}_{P}\left[\left(2^{q}\left(\frac{S_{n}}{\sqrt{n}}\right)^{q}-\alpha\right)_{+}\right]$

where $S_{n}=\sum_{j=1}^{n} U_{j}$. Let $N$ be a centered and reduced normal variable, according to the central limit theorem

$$
P\left(\frac{\left|S_{n}\right|}{\sqrt{n}} \geq \frac{\alpha^{\frac{1}{q}}}{2}\right) \underset{n \rightarrow \infty}{\rightarrow} P\left(|N| \geq \frac{\alpha^{\frac{1}{q}}}{2}\right) .
$$

Moreover, since $U_{1} \in L^{q}(W)$, the central limit theorem remains valid for the Wasserstein distance of order $q$ (see for example [15]). It follows that the random variables $\left(\frac{S_{n}}{\sqrt{n}}\right)^{q}$ are uniformly integrable, thus,

$$
\mathbb{E}_{P}\left[\left(2^{q}\left(\frac{S_{n}}{\sqrt{n}}\right)^{q}-\alpha\right)_{+}\right] \underset{n \rightarrow \infty}{\rightarrow} \mathbb{E}_{P}\left[\left(2^{q} N^{q}-\alpha\right)_{+}\right] .
$$


Hence

$$
\lim _{n} \sup A_{n, \alpha} \underset{\alpha \rightarrow \infty}{\longrightarrow} 0 . \square
$$

Applying the preceding proposition to (15) one has

Proposition 4.0.6. If $h: \mathbb{R}^{p} \rightarrow \mathbb{R}$ is given by a multimeasure and if $\left(U_{1}, U_{1}^{\#}\right) \in$ $L^{2 p}(W) \times L^{2 p}(W \otimes \hat{W})$ then $\phi_{h}\left(X_{n}\right)$ converges in Dirichlet law toward $\phi_{h}{ }_{*} S_{O U}$.

Remark 10: We can show that the preceding results extend in the framework of the functional Lindeberg-Feller theorem ([10], p.226). More precisely, let $\left(U_{k}\right)$ be a sequence of centered and normalized i.i.d random variables and $\left(a_{n, k}\right)$ a triangular array of real numbers such that

a) There exists an increasing function $a:[0,1] \rightarrow \mathbb{R}$ of classe $C^{1}$, null at zero such that $\forall t \in[0,1]$,

$$
\frac{1}{n} \sum_{k=1}^{[n t]} a_{n, k}^{2} \underset{n \rightarrow \infty}{\rightarrow} a(t)
$$

b) There exists a constant $K$ such that $\forall n \in \mathbb{N}^{*}, \forall k \in\{0, \ldots, n\},\left|a_{n, k}\right| \leq K$.

The results of sections 3 and 4 remain valid with the process

$$
Z_{n}(t)=\frac{1}{\sqrt{n}}\left(\sum_{k=1}^{[n t]} a_{n, k} U_{k}+(n t-[n t]) a_{n,[n t]+1} U_{[n t]+1}\right)
$$

instead of $X_{n}$ and $S_{O U}^{\sqrt{a^{\prime}} h}$ instead of $S_{O U}^{h} \bullet$

\section{REFERENCES}

1. E. Alòs, O. Mazet, D. NuAlart : Stochastic calculus with respect to Gaussian processes, Ann Proba. 29, no. 2, 766-801, 2001.

2. X. Bardina, M. Jolis : Weak convergence to the multiple Stratonovich integral, Stochastic Process. Appl. 90, no. 2, 277-300, 2000.

3. P. Billingsley : Convergence of probability measures, John Wiley \& Sons Inc., 1999.

4. N. Bouleau : Théorème de Donsker et formes de Dirichlet, Bull. Sci. Math. 129, no. 5, 369-380, 2005.

5. N. Bouleau, C. Chorro : Error structures and parameter estimation, C. R. Acad. Sci. Paris, Ser. I 338, 305-310, 2004.

6. N. Bouleau : Error calculus for finance and physics: The language of Dirichlet forms, de Gruyter expositions in mathematics 37, 2003.

7. N. Bouleau, F. Hirsch : Dirichlet forms and analysis on Wiener space, de Gruyter studies in mathematics 14, 1991.

8. C. Chorro : On an extension of the central limit theorem to Dirichlet forms, Cahiers de la mse, 2004.80, Univ. Paris 1, 2004. 
9. R. Delgado, M. Jolis : Weak approximation for a class of gaussian processes, J. Appl. Probab. 37, no. 2, 400-407, 2000.

10. D. Dacunha-Castelle, M. Duflo : Probabilités et statistiques 2, Masson, 1993.

11. D.Feyel, A. De La Pradelle : Espaces de Sobolev Gaussiens, Ann. Institut Fourier 39, no. 4, 875-908, 1989.

12. M. Fukushima : Dirichlet forms and Markov processes, North-Holland mathematical library $23,1980$.

13. J. JACOD, A.N. ShIRYAev : Limit theorems for stochastic processes, Springer-Verlag, 1987.

14. A. Jakubowski, J. Mémin, G. Pagès : Convergence en loi des suites d'intégrales stochastiques sur l'espace $D^{1}$ de Skorokhod, Probab. Theory Related Fields 81, 111-137, 1989.

15. O. Jonhson, R. Samworth : Central limit theorem and convergence to stable laws in Mallows distance, Preprint, 2004.

16. T.G. Kurtz, P. Protter: Weak limit theorems for stochastic integrals and stochastic differential equations, Ann. Probab. 19, no. 3, 1035-1070, 1991.

17. Z. MA, M. RöcKNER : Introduction to the theory of (non-symmetric) Dirichlet forms, Springer-Verlag, 1992.

18. D. NuAlart : Malliavin calculus and related topics, Springer-Verlag, 1995.

19. D. NuAlart, M. ZAKAI : Multiple Wiener-Ito integrals possessing a continuous extension, Probab. Theory Related Fields 85, no. 1, 131-145, 1990.

20. A.W. VAn Der VAart, J.A. Wellner : Weak convergence and empirical processes, Springer-Verlag, 1996. 